\title{
Inverse magnetoelectric effect in disk samples of ferrite-piezoelectric composites
}

\author{
D. A. Filippov ${ }^{1}$, T. A. Galkina ${ }^{1}$, V. M. Laletin ${ }^{2}$ \\ ${ }^{1}$ Novgorod State University, Russia, Velikii Novgorod \\ ${ }^{2}$ Institute of Technical Acoustics, Belarus, Vitebsk
}

Email address:

Dmitry.Filippov@novsu.ru (D. A. Filippov), taisiya_galkina@mail.ru (T. A. Galkina), laletin57@rambler.ru (V. M. Laletin)

To cite this article:

D. A. Filippov, T. A. Galkina, V. M. Laletin. Inverse Magnetoelectric Effect in Disk Samples of Ferrite-Piezoelectric Composites. Science Research. Vol. 1, No. 2, 2013, pp. 12-18. doi: 10.11648/j.sr.20130102.11

\begin{abstract}
A theory of the inverse magnetoelectric (ME) effect in the region of electromechanical resonance (EMR) for the samples in the form of a disk is presented. The expression for the coefficient of inverse ME-conversion on the effective parameter method is derived. It is shown that observed the peak increase of effect in the region of electromechanical resonance, resonance frequencies are differed for direct and inverse magnetoelectric effect. Resonance frequencies dependence on ferrite percentage for ferrite-nickel spinel and lead zirconate titanate samples are investigated experimentally.
\end{abstract}

Keywords: Inverse Magnetoelectric Effect, Electromechanical Resonance

\section{Introduction}

The magnetoelectric (ME) effect is relate to cross effects and consists in that polarization appears in a sample under the action of a magnetic field (direct ME effect) and, vice versa, magnetization appears upon the application of an electric field (inverse ME effect). This effect which was originally predicted by Dzyaloshinskii [1] and then observed in experiments [2,3], is of interest not only for basic science, but for applications as well, since it couples the values, that has different tensor dimensions: polarization (polar vector) with magnetizing intensity (axial vector), and, vice versa, magnetization (axial vector) with electric intensity (polar vector). Presence of the ME effect in monocrystal directly coupled with crystal symmetry [4], and its arising is ride by joint action of spin-orbit interaction, odd part of intracrystalline field and external electric field [5]. ME effect in the ferrite-piezoelectric composite is individually absent in ferrite and in piezoelectric phases. Its arising is condition on mechanical interaction between magnetostriction and piezoelectric subsystems. In the ferrite component, mechanical stresses arise due to magnetostriction in a magnetic field, that are transferred to the piezoelectric phase, where occur an electric polarization. And, vice versa, for the inverse effect, deformations induced in the piezoelectric phase under the action of an electric field are transferred to the ferrite phase, where the magnetostriction leads to magnetization of the sample.
Presently, the direct ME effect is known in much detail [6], whereas the inverse effect is still insufficiently studied and even the number of publications on this phenomenon is very restricted [7-10]. In this works inverse ME effect investigated experimentally for the samples in the form of a plate, where was used lead zirconate titanate (PZT) piezoceramic as the piezoelectric, and materials with large magnetostriction coefficient as the ferrite, to be exact: in [7] - D-Terfenol (three-layer structure D-Terfenol-PZT-D- Terfenol), in [8] nickel (three-layer structure PZT-Ni-PZT) and in $[9,10]$ galfenol (two-ply structure galfenol-PZT). In the present works realized the measurement of coefficient of inverse ME-conversion frequency dependence and experimentally discovered, that this dependence has resonant nature, in a similar as ME voltage coefficient frequency dependence for the direct effect. However detailed theoretical description of the inverse ME effect it not realized in this works. In work [11] it is shown the theoretical description of the inverse ME effect for the samples in the form of a plate. However the samples in the form of a disk are much often used in practice, but not in the form of a plate. But data of work [11] for the samples in the form of a disk can not be used. Detailed theoretical description of the inverse ME effect and experimental results for the ferrite-nickel spinel and lead zirconate titanate samples in the form of a disk are presented in this work.

\section{Theory of Effect}




\subsection{Longitudinal Effect}

Effective parameter method was got wide spread occurrence at the description of physical phenomenon in the ferrite-piezoelectric composites [12, 13]. This method consists in that composition material from the macroscopic point of view considered as a homogeneous medium with some effective parameters, which are found by combined solved equations of elasto- and electrodynamics for the ferrite and piezoelectric phases with following averaging. Apparently that this method can be used, when the characteristic dimensions of composite building blocks much smaller than sound-wave-length. As the characteristic dimensions of the building blocks in the typical composition materials are order the decade of micron, that this description method can be used as far as frequency order by a hundred $\mathrm{MHz}$.

Consider a model system comprising a sample of the ferrite-piezoelectric composite material in the form of a thin disk, with the sample radius $\mathrm{R}$ and thickness $\mathrm{d}$. The thin metal electrodes have been applied on the top and on the bottom surfaces of the disk, and on which the side face an induction coil, with $N$ numbers of turns (fig.1). Let the sample is polarized transversely to contacts plane (axis Z). Direct magnetic field directs either along the samples polarization (longitudinal effect).

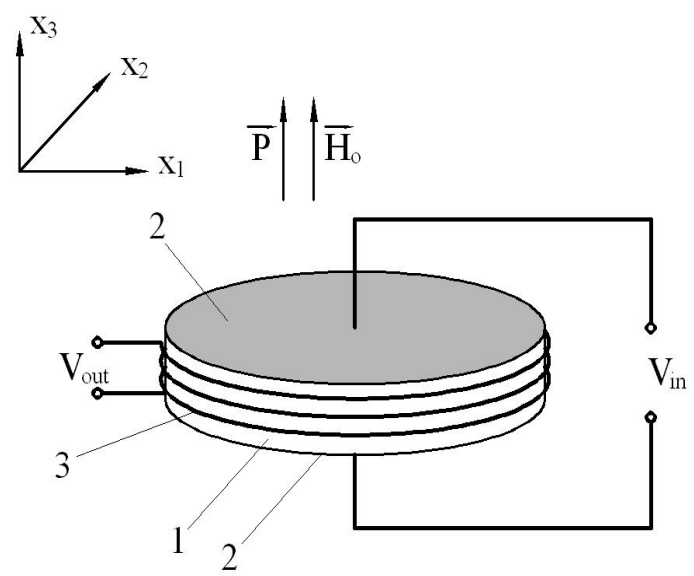

Figure 1. Schematic diagram of the structure with: (1) sample; (2) metal electrodes; (3) coil with $N$ numbers of turns.

Alternating electric field with frequency $\omega$, that given on the sample, is give rise to oscillation in the sample, which are spread along the sample surface - is the radial oscillation, and all along sample thickness - is the thicknesses oscillation. In future we will come to nothing more than the consideration of the most low-frequency radial oscillation.

Let us assume that the dick is thin, i.e. $\mathrm{d}<<\mathrm{R}$.. That the surfaces of the composite dick are free that is, the normal components of the stress tensor on these surfaces are vanishing. Since the dick is thin, we can assume that $\mathrm{T}_{3}=0$ not only on the surface, but in the volume of the dick. Accordingly, the equations for components of the strain tensor $\mathrm{Si}$ and magnetic induction $B_{3}$ can be written as follows:

$$
\begin{array}{r}
S_{1}=s_{11} T_{1}+s_{12} T_{2}+d_{31} E_{3}+q_{31} H_{3}, \\
S_{2}=s_{12} T_{1}+s_{11} T_{2}+d_{31} E_{3}+q_{31} H_{3}, \\
B_{3}=\mu_{33} H_{3}+q_{31}\left(T_{1}+T_{2}\right) .
\end{array}
$$

where $s_{i j}$ is the effective compliance of the composite, $d_{i j}$ and $\mathrm{q}_{\mathrm{ij}}$ is the effective piezoelectric and piezomagnetic components, $\varepsilon_{\mathrm{ij}}$ is the effective permittivity, $\mathrm{E}_{\mathrm{i}}$ and $\mathrm{H}_{\mathrm{i}}$ is the projections of the tensity vectors of variable electric and magnetic fields. Design procedure of effective parameters of composition material is presented in $[14,15]$.

It is convenient to used the symmetry of a problem and turn to the cylindrical co-ordinates $\mathrm{z}, \mathrm{r}$ and $\theta$, using the conversions presented in [16] for further calculations. The axial symmetry results in nonzero components of the pressure and strain tensors $\mathrm{T}_{\mathrm{rr}}, \mathrm{T}_{\theta \theta}, \mathrm{S}_{\mathrm{rr}}$ and $\mathrm{S}_{\theta \theta}$. Others components of the pressure and strain tensors equal to zero. Besides from the axial symmetry results, that the displacement component $\mathrm{u}_{\theta}$ equal to zero.

We allowance for this, the above expression (1-3) reduces to

$$
\begin{gathered}
S_{r r}=s_{11} T_{r r}+s_{12} T_{\theta \theta}+d_{31} E_{3}+q_{31} H_{3}, \\
S_{\theta \theta}=s_{12} T_{r r}+s_{11} T_{\theta \theta}+d_{31} E_{3}+q_{31} H_{3}, \\
B_{3}=\mu_{33} H_{3}+q_{31}\left(T_{r r}+T_{\theta \theta}\right) .
\end{gathered}
$$

The equation of motion of the medium for the radial oscillation reduces to

$$
\frac{\partial T_{r r}}{\partial r}+\frac{1}{r}\left(T_{r r}-T_{\theta \theta}\right)+\rho \omega^{2} u_{r}=0
$$

where $\rho$ is the density of composite.

Evaluate from $(4,5)$ components of stress tensor through components of pressure tensor, as result we get:

$$
\begin{gathered}
T_{r r}=\frac{1}{S_{11}\left(1-v^{2}\right)}\left(S_{r r}+v S_{\theta \theta}-(1+v)\left(d_{31} E_{3}+q_{31} H_{3}\right)\right), \\
T_{\theta \theta}=\frac{1}{S_{11}\left(1-v^{2}\right)}\left(v S_{r r}+S_{\theta \theta}-(1+v)\left(d_{31} E_{3}+q_{31} H_{3}\right)\right),
\end{gathered}
$$

where $v=-s_{12} / s_{11}$ is the Poisson's ratio.

In order to take the equation for the radial displacement, substitute $(8,9)$ into equation of motion of the medium (7). After the transformations the equation (7) is reduced to Bessel equation

$$
\frac{\partial^{2} u_{r}}{\partial r^{2}}+\frac{1}{r} \frac{\partial u_{r}}{\partial r}-\frac{u_{r}}{r^{2}}+k^{2} u_{r}=0
$$

where $k=\sqrt{\rho s_{11}\left(1-v^{2}\right)} \omega$. General solution of the (10) can be presented as superposition of the first and second order Bessel functions

$$
u_{r}=c_{1} J_{1}(k r)+c_{2} Y_{1}(k r) \text {. }
$$


Constants $c_{1}$ and $c_{2}$ are defined by the boundary conditions: the displacements are lacking in the disk centre, i.e. at $r=0$ displacement $u_{r}=0$, but the normal strains equal to zero on side surfaces, therefore at $r=R$ strain $T_{r r}=0$. This is give for constants following expressions [17]:

$$
c_{2}=0, c_{1}=\frac{(1+v) R}{\kappa J_{0}(\kappa)-(1-v) J_{1}(\kappa)}\left(q_{31} H_{3}+d_{31} E_{3}\right) .
$$

Here introduced nondimensional variable $\kappa=k R$, which values are depends on frequency, disk radius and rate of elastic oscillation propagation.

Substituting the values of constants $c_{1}$ and $c_{2}$ in (11) and, expressing the deformation through the medium displacement, we get expressions for the components of the strain tensors

$$
\begin{gathered}
T_{r r}=\frac{1}{s_{11}(1-v)}\left[\frac{\kappa J_{0}(k r)-(1-v) \frac{R}{r} J_{1}(k r)}{\kappa J_{0}(\kappa)-(1-v) J_{1}(\kappa)}-1\right] \cdot\left(q_{31} H_{3}+d_{31} E_{3}\right)(13) \\
T_{\theta \theta}=\frac{1}{s_{11}(1-v)}\left[\frac{v \mathcal{N} J_{0}(k r)+(1-v) \frac{R}{r} J_{1}(k r)}{\kappa J_{0}(\kappa)-(1-v) J_{1}(\kappa)}-1\right] \cdot\left(q_{31} H_{3}+d_{31} E_{3}\right)(
\end{gathered}
$$

The magnetization, which is appeared in the sample as a result of magnetostriction as a result of medium mechanical deformation, we define from Eq. (6). Substituting the expressions (13) and (14) in equation (6) we get:

$$
B_{3}=\mu_{33} H_{3}+\frac{q_{31}}{s_{11}(1-v)}\left[\frac{(1+v) \kappa J_{0}(k r)}{\kappa J_{0}(\kappa)-(1-v) J_{1}(\kappa)}-2\right] \cdot\left(q_{31} H_{3}+d_{31} E_{3}\right)
$$

For the experimental investigation of inverse ME effect, it is measured the EMF of induction, that originated in coil at the magnetic flux measuring, due to the change of the magnetization of capacitor magnetoelectric dielectric. Usually, the voltmeter resistance in these measurements is much greater than the coil resistance at the experiment, we can assume that the open-circuit condition is valid $(\mathrm{I}=0)$ and, hence, $\mathrm{H}_{3}=0$. We allowance for this, the above expression (15) for the magnetic induction reduces to:

$$
B_{3}=\frac{q_{31} d_{31}}{s_{11}(1-v)}\left[\frac{(1+v) \kappa J_{0}(k r)}{\kappa J_{0}(\kappa)-(1-v) J_{1}(\kappa)}-2\right] \cdot E_{3} .
$$

The inverse ME conversion factor for the longitudinal configuration of fields is defined as $\alpha_{B, L}=\left\langle B_{3}\right\rangle / E_{3}$, where $\left\langle B_{3}\right\rangle$ is the average magnetic induction in the sample that is determined as . $\left\langle B_{3}\right\rangle=\frac{1}{\pi R^{2}} \cdot \int_{0}^{2 \pi} d \theta \int_{0}^{R} B_{3} r d r$

Calculating the average magnetic induction and substituting this expression into the above definition, we eventually obtain the following expression:

$$
\alpha_{B, L}=\frac{2 q_{31} d_{31}}{s_{11}(1-v)} \cdot\left[\frac{(1+v) J_{1}(\kappa)}{\Delta_{r}}-1\right]
$$

where

$$
\Delta_{r}=\kappa J_{0}(\kappa)-(1-v) J_{1}(\kappa) .
$$

The EMF induced in the coil by magnetic flux is

$$
\varepsilon_{i n}=-\frac{\partial \Phi_{L}}{\partial t}=-N \frac{\partial}{\partial t} \int_{0}^{2 \pi} d \theta \int_{0}^{R} B_{3} r d r=i \omega N \pi R^{2} \alpha_{B, L} \cdot E_{3} .
$$

Supposing, that all absorbed voltage take place on the measuring voltmeter, and the electric field strength in sample bound up with the input voltage by $E_{3}=V_{i n} / d$, and for the voltage ratio (transformer coefficient) $k_{L}=V_{\text {out }} / V_{\text {in }}$ for the longitudinal ME effect is

$$
k_{L}=\omega N \frac{\pi R^{2}}{d} \alpha_{B, L}
$$

Accordingly, this structure can be used as a transformer, which has only one winding in contrast to traditional type transformer.

\subsection{Transverse Effect}

For the transverse ME effect direct magnetic field $\vec{H}_{0}$ in the sample directs either along the samples polarization $\vec{P}$. Applied to the contacts electrical field to provoke the mechanical oscillation in the sample, and change the magnetization in all along sample thickness at the result. For the experimental investigation of transverse ME effect we use the structure represented at fig. 2 .
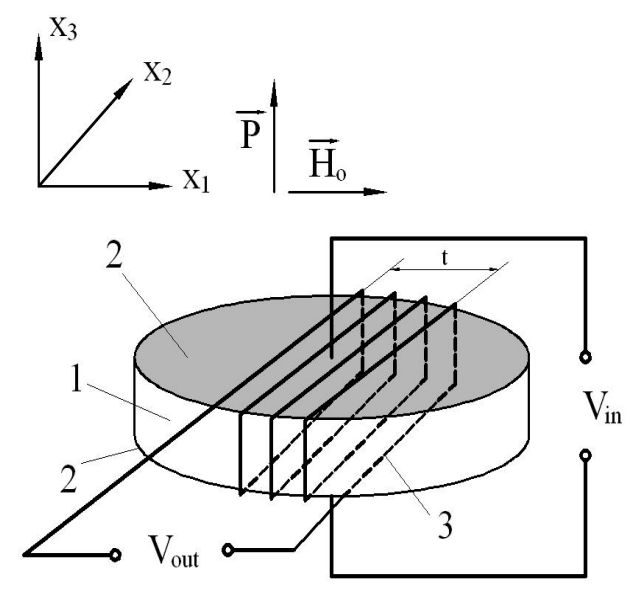

Figure 2. Schematic diagram of the structure with: (1) sample; (2) metal electrodes; (3) coil with $N$ numbers of turns, $t$-coil thickness.

Equations (1-3) for the pressure tensor $\mathrm{Si}$ and for the magnetic induction $B_{1}$ for the transverse ME effect takes form:

$$
\begin{array}{r}
S_{1}=s_{11} T_{1}+s_{12} T_{2}+d_{31} E_{3}+q_{11} H_{1}, \\
S_{2}=s_{12} T_{1}+s_{11} T_{2}+d_{31} E_{3}+q_{12} H_{1},
\end{array}
$$




$$
B_{1}=\mu_{11} H_{1}+q_{11} T_{1}+q_{12} T_{2} .
$$

Components of the strain tensors expression are get complicated by the direct magnetic field $H_{1}$ break its system axial symmetry at the turn to the cylindrical co-ordinates. However, taking into account, that in the experiment used the open circuit condition and alternating magnetic field strength equal to zero, the medium oscillation are provoke by alternating electric field, directed along axes $X_{3}$, as for the longitudinal effect. Equation of medium motion for radial oscillation reduced to (10), which solution given the components of the strain tensors:

$$
\begin{aligned}
& T_{r r}=\frac{1}{s_{11}(1-v)}\left[\frac{\kappa J_{0}(k r)-(1-v) \frac{R}{r} J_{1}(k r)}{\kappa J_{0}(\kappa)-(1-v) J_{1}(\kappa)}-1\right] \cdot d_{31} E_{3}, \\
& T_{\theta \theta}=\frac{1}{s_{11}(1-v)}\left[\frac{v \kappa J_{0}(k r)+(1-v) \frac{R}{r} J_{1}(k r)}{\kappa J_{0}(\kappa)-(1-v) J_{1}(\kappa)}-1\right] \cdot d_{31} E_{3} .
\end{aligned}
$$

Components of the strain tensors $T_{1}$ and $T_{2}$ connected with components $T_{r r}$ and $T_{\theta \theta}$ by:

$$
\begin{array}{r}
T_{1}=T_{r r} \cos ^{2} \theta+T_{\theta \theta} \sin ^{2} \theta, \\
T_{2}=T_{r r} \sin ^{2} \theta+T_{\theta \theta} \cos ^{2} \theta,
\end{array}
$$

Using this ratios, for the magnetic induction in the cylindrical co-ordinates we get:

$$
B_{1}=T_{r r}\left(q_{11} \cos ^{2} \theta+q_{12} \sin ^{2} \theta\right)+T_{\theta \theta}\left(q_{11} \sin ^{2} \theta+q_{12} \cos ^{2} \theta\right) .
$$

In a similar to longitudinal effect, inverse $\mathrm{ME}$ conversion factor is defined as $\alpha_{B, T}=\left\langle B_{1}\right\rangle / E_{3}$ for the transverse effect. Substituting expressions (23) and (24) in equation (28), and calculating the average magnetic induction $\left\langle B_{1}\right\rangle$, we obtain the following expression for inverse ME conversion factor:

$$
\alpha_{B, T}=\frac{\left(q_{11}+q_{12}\right) d_{31}}{s_{11}(1-v)} \cdot\left[\frac{(1+v) J_{1}(\kappa)}{\Delta_{r}}-1\right],
$$

where $\Delta_{r}$ defined by (18).

For the defined of EMF induced in the coil, we limited by the case, when coil thickness $t$ is smaller then disk radius $R$. At this approximation we can take, that magnetic flux, splitting the coil equal to $\Phi_{T}=N \cdot 2 R \cdot d$ and for transformer coefficient:

$$
k_{T}=\omega N \cdot 2 R \cdot \alpha_{B, T} .
$$

\section{Results Discussion Comparison with Experiment}

As following from expression (17) and (29) for inverse $\mathrm{ME}$ conversion factor its value in proportion to product of values of piezoelectric $d_{31}$ and piezomagnetic $q_{i 1}$ modules and inversely to compliance module $s_{11}$ value. However for the transverse effect the value of coefficient $\alpha_{B, T}$ is proportional to product $\left(q_{11}+q_{12}\right) d_{31}$. And for the longitudinal effect the value $\alpha_{B, L}$ is proportional to product $q_{31} d_{31}$. As $\left(q_{11}+q_{12}\right)>q_{31}$, that the value of the transverse effect bigger, than of longitudinal.

Frequency dependence of the inverse ME conversion factor as the frequency dependence of the ME voltage coefficient $\alpha_{E}$ for the direct effect, has the resonance nature. At the low frequency region, when parameter $\kappa<<1$, the value of the inverse ME conversion factor practically is not depend on frequency. However at the frequency, that corresponds to condition $\Delta_{r}=0$, it is observe the peak increase of coefficient. Roots of these equation take the name resonant frequencies $f_{\text {res }}$. The value of lower resonant frequency to average about $f_{\text {res }} \approx 300 \mathrm{kHz}$ for the ferrite-nickel spinel and lead zirconate titanate samples with radius nearly $R \approx 5 \mathrm{~mm}$.

It should be noted that, in contrast to the inverse ME effect, the resonant growth $\alpha_{E}=<E>/ H$ in the direct $\mathrm{ME}$ effect is observed at an antiresonance frequency $f_{\text {anres }}$ that corresponds to the condition $\Delta_{a}=0$, where

$$
\Delta_{a}=1-K_{p}^{2}+K_{p}^{2}(1+v) J_{1}(\kappa) / \Delta_{r} .
$$

Where $K_{p}^{2}=2 d_{31}^{2} / \varepsilon_{33} s_{11}(1-v) \quad-$ is the square coefficient of electromechanical coupling for radial oscillations.

There are inverse ME conversion factor frequency dependences for the ferrite-nickel spinel and lead zirconate titanate samples in the form of a disk calculated by (17) and (29) resulted in the pictures 3 and 4 for the transverse and longitudinal effects. Sample radius $\mathrm{R}=1 \mathrm{sm}$.

The following quantity values used at the calculations: Ferrite:

$$
\begin{aligned}
& s_{11}^{m}=6.8 \cdot 10-12 \mathrm{~m} 2 / \mathrm{N}, \quad s_{12}^{m}=2.4 \cdot 10-12 \mathrm{~m} 2 / \mathrm{N}, \\
& q^{m}{ }_{11}=-1880 \cdot 10-12 \mathrm{~m} / \mathrm{A}, \quad q^{m}{ }_{12}=320 \cdot 10-12 \mathrm{~m} / \mathrm{A}, \\
& q_{31}^{m}=556 \cdot 10-12 \mathrm{~m} / \mathrm{A}, \quad \rho^{m}=5200 \mathrm{~kg} / \mathrm{m} 3 ; \\
& \text { Piezoelectric: } \\
& s_{11}^{p}=15.3 \cdot 10-12 \mathrm{~m} 2 / \mathrm{N}, \quad s_{12}^{p}=5.4 \cdot 10-12 \mathrm{~m} 2 / \mathrm{N}, \\
& d^{p}{ }_{31}=175 \cdot 10-12 \mathrm{~m} / \mathrm{V}, \quad \rho^{p}=7800 \mathrm{~kg} / \mathrm{m} 3 .
\end{aligned}
$$

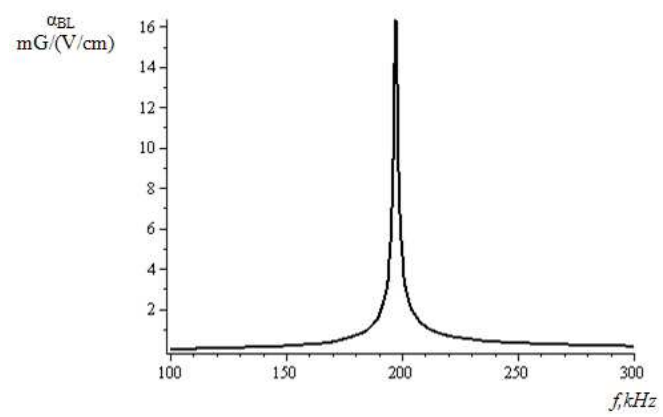

Figure 3. Inverse ME conversion factor frequency dependences for longitudinal effects 


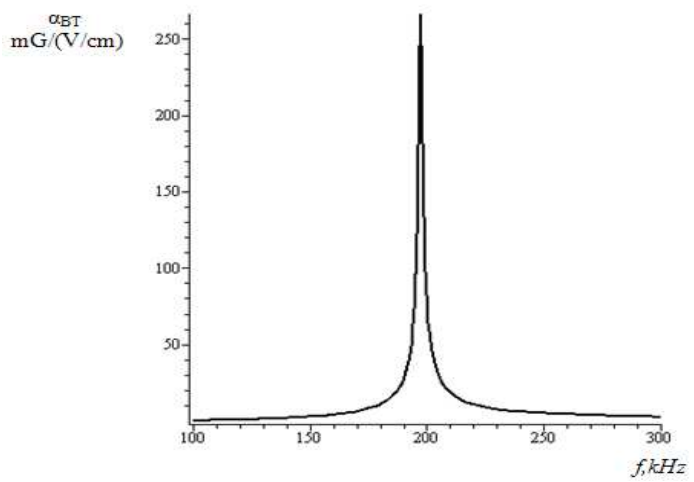

Figure 4. Inverse ME conversion factor frequency dependences for transverse effects

Frequencies of resonance and antiresonance has at least the closely values, but they are distinguishable experimentally.

Experimental investigations of effect were realized for the samples of ferrite-nickel spinel and PZT. Samples has a disk form with radius $R=4.5 \mathrm{~mm}$. Before measurement the samples was polarized by electric field with strength 4 $\mathrm{kV} / \mathrm{mm}$ during two hours at the temperature $80^{\circ} \mathrm{C}$. It is used method, based on measuring of alternating voltage, that appearing at the superposition of alternating and slowly changing magnetic field on the sample, for the investigations of direct ME effect. For the investigations of inverse ME effect we used method, based on measuring of alternating voltage, that appearing at the measuring coil when it place in slowly changing magnetic field and when the alternating electric field apply to the sample. At first was investigated the field dependence of low-frequency $\mathrm{ME}$ signal. ME effect dependence of bias field strength was measured at the constant value of alternating magnetic field strength 1 Oe. Then was investigated frequency dependence of magnetoelectric coefficient in the electromechanical resonance region at the bias field strength, that agree with a maximum of effect. The open circuit condition realized very well at the measurement. Experimental data are shown in table 1 .

Table 1. Table information

\begin{tabular}{lllllll}
\hline Ferrite percentage, $\%$ & $\mathbf{1 0}$ & $\mathbf{2 0}$ & $\mathbf{3 0}$ & $\mathbf{4 0}$ & $\mathbf{5 0}$ & $\mathbf{6 0}$ \\
\hline $\mathrm{f}_{\text {res }}, \mathrm{kHz}$ & 39,9 & 13,4 & 34,8 & 59,7 & 78,8 & 72,0 \\
$\mathrm{f}_{\text {anres }}, \mathrm{kHz}$ & 11,9 & 27,9 & 42,6 & 53,4 & 30,5 & 72,5 \\
$\Delta \mathrm{f}, \mathrm{kHz}$ & 2,0 & 4,5 & 8 & 7 & 7 & 5 \\
$\alpha_{B, T}\left(f=f_{\text {res }}\right), \mathrm{mG} \cdot \mathrm{cm} / \mathrm{V}$ & 30 & 20 & 10 & 72 & 49 & ) \\
$\alpha_{E, T}\left(f=f_{\text {anres }}\right), \mathrm{V} /(\mathrm{cm} \cdot \mathrm{Oe})$ & 4 & 34 & 57 & 54 & 39 & 52 \\
$\alpha_{B, T}(f=1 \mathrm{kHz}), \mathrm{mG} \cdot \mathrm{cm} / V$ & 1161 &, 227 & .194 &, 162 &, 117 & 065 \\
$\alpha_{E, T}(f=1 \mathrm{kHz}), \mathrm{mV} /(\mathrm{cm} \cdot \mathrm{Oe})$ & 1 & 2 & 5 & 7 & 39 & 7 \\
\hline
\end{tabular}

As following from experiment, in full accordance with theory, there is a peak increase of effect at the resonant and antiresonant frequency. This frequencies values defining by parameter $\kappa=k R=\omega \sqrt{\rho s_{11}\left(1-v^{2}\right)} R$, at which the values of expressions (18) and (31) for the value $\Delta_{r}$ for inverse effect and for the value $\Delta_{a}$ for direct effect are equal to zero. For the Poisson's coefficient $v=0.34$ the equality to zero of these expressions is happen when value $\kappa_{0}=2.074$. This implyies, that the resonant frequency defining by condition $f_{\text {res }}=2.074 /\left(2 \pi \sqrt{\rho s_{11}\left(1-v^{2}\right)} R\right)$. Piezoceramic density PZT $\quad{ }^{p} \rho=7800 \mathrm{kG} / \mathrm{m}^{3} \quad, \quad$ compliance module ${ }^{p} S_{11}=15.3 \cdot 10^{-12} \mathrm{~m}^{2} / \mathrm{N}, \quad$ ferrite-nickel spinel density ${ }^{m} \rho=5200 \mathrm{kG} / \mathrm{m}^{3} \quad, \quad$ its compliance module ${ }^{m} s_{11}=6.5 \cdot 10^{-12} \mathrm{~m}^{2} / \mathrm{N}$. With increase of the ferrite percentage it is occur of composite effective density decrease and composite effective compliance decrease, as result to increase of resonant and antiresonant frequency. Resonant and antiresonant frequency's difference defining by the coefficient of electromechanical coupling $K_{p}$, as easily to see by expressions (18) and (31) for $\Delta_{r}$ and for $\Delta_{a}$. Really, supposing $f_{\text {anres }}=f_{\text {res }}+\Delta f$ and expanding expression for $\Delta_{a}$ by small parameter $\Delta \kappa=\Delta f 2 \pi \sqrt{\rho s_{11}\left(1-v^{2}\right)} R$ for relative difference of frequencies we get:

$$
\frac{\Delta f}{f_{\text {res }}} \approx \frac{(1+v) K_{p}^{2}}{\kappa_{0}^{2}\left(1-(1-v) / \kappa_{0}^{2}-v J_{0}\left(\kappa_{0}\right) /\left(\kappa_{0} \cdot J_{1}\left(\kappa_{0}\right)\right)\right)} .
$$

Or, substituting the numerical values $v=0.34$ and $\kappa_{0}=2.074$, for relative difference of frequencies we get the resultant expression in the following form:

$$
\frac{\Delta f}{f_{\text {res }}} \approx 0.4 \cdot K_{p}^{2} \text {. }
$$

With increase of the ferrite percentage, the coefficient of electromechanical connection increasing in consequence of composite effective piezomodule value increase, as a result at the increase of resonant and antiresonant frequency difference.

The experimental and theoretical frequency dependences of inverse ME conversion factor $\alpha_{B}$ for PZT(23-1) - 70 mass.\%, NiFe1,9Co0,01O4 - 30 mass.\% structure represented at fig.5. The experimental measuring realized when value of bias field $\mathrm{Hm}=380 \mathrm{Oe}$, that meet the maximum of ME interaction.

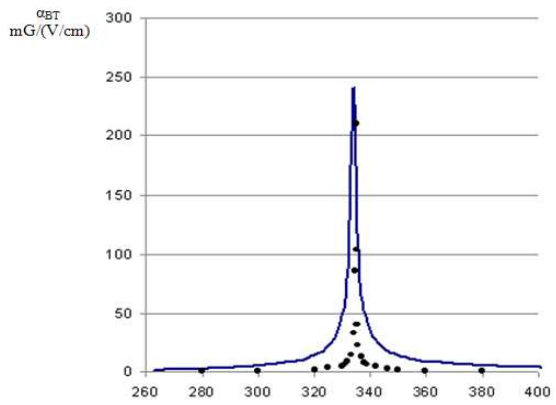

$f, k H z$

Figure 5. Inverse ME conversion factor $\alpha_{B}$ frequency dependences for transverse effects for PZT(23-1) - 70 mass. \%, NiFe1,9Co0,01O4-30 mass.\% structure, where the full line is the theoretical dependence, points are the experimental data 
In this case the inverse ME signal amplification occurring at the frequency $f_{\text {res }}=335 \mathrm{kHz}$, and the inverse ME conversion factor resonant value three times as many as its low-frequency value. In comparison with multilayer composites, this good quality accounted for the applied of piezoelectric with high good quality $(Q \approx 3000)$ and the selection of operating practices, that resulting to performance of the mechanical contact between splinters. There is a similar effect was observed on the samples with other structures, but the resonance frequencies are vary depending on composite percentage composition vary.

The experimental and theoretical resonant frequency dependences of ferrite content in composite are represented at fig. 6 .

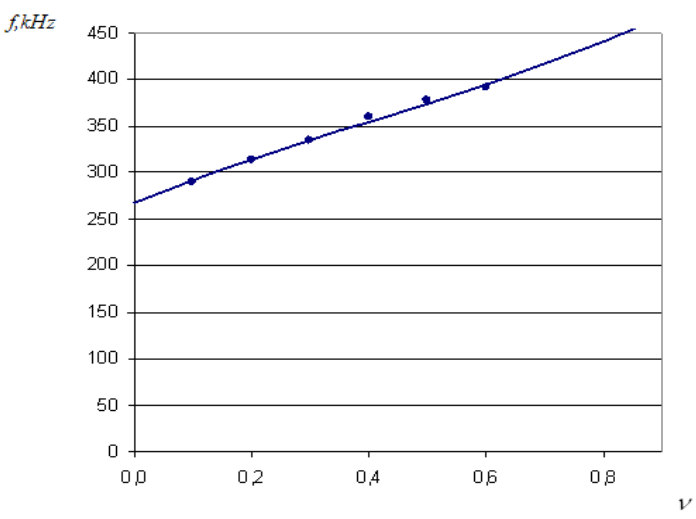

Figure 6. Resonance frequency dependences of ferrite content in composite, where the full line is the theoretical dependence, points are the experimental data

As appears from the fig. 6, with increase of ferrite percentage there is a resonant frequency increase. This account for composite effective density and effective compliance decrease by ferrite percentage increase, that resulting in resonant frequency increase.

The resonance and antiresonance frequencies dependences of ferrite content in composite are represented at fig. 7 .

The difference of resonance and antiresonance frequencies represented at fig. 8 .

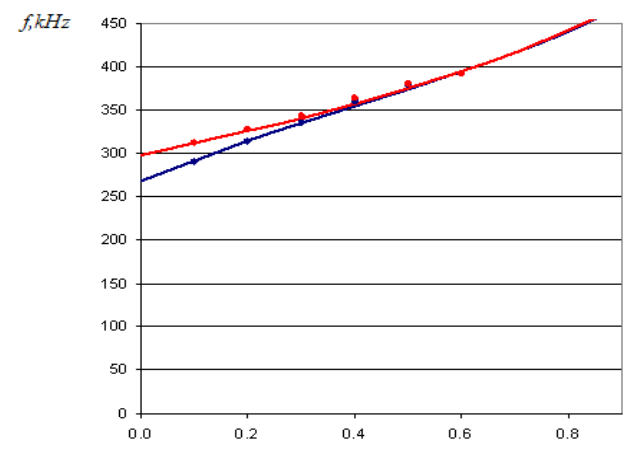

Figure 7. Resonance (the bottom characteristic) and antiresonance (the upper characteristic) frequencies dependences of ferrite content in composite, where the full line is the theoretical dependence, points are the experimental data

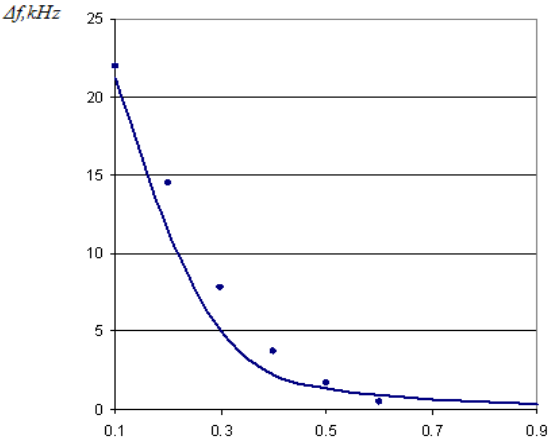

Figure 8. The difference of resonance and antiresonance frequencies, where the full line is the theoretical dependence, points are the experimental data

With increase of the ferrite percentage by piezomodule effective value decrease and decrease of electromechanical coupling factor, as a resulted to decrease of resonance and antiresonance frequencies.

Effect of composite structure on the inverse ME conversion factor $\left(f_{\text {res }}=335 \mathrm{kHz}\right)$ represented at fig.9. We plotting of inverse ME conversion factor dependence of ferrite inclusion volume fraction by using the theoretical model.

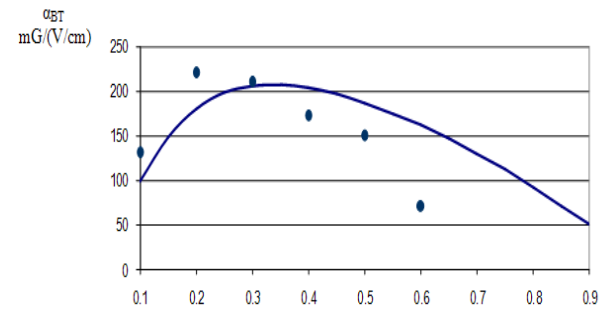

Figure 9. Dependence of inverse ME conversion factor by ferrite inclusion volume fraction ( $f_{\text {res }}=335 \mathrm{kHz}$ ), where the full line is the theoretical dependence, points are the experimental data

As can be seen by diagram (fig. 9), the dependence of inverse ME conversion factor by ferrite inclusion volume fraction has a maximum. The inverse ME conversion factor $\alpha_{B}$ is proportional to product of effective values of piezoelectric and piezomagnetic modules and inversely proportional to module of composite compliance. With ferrite percentage increase there is piezomodule decrease, that reducing to decrease of $\alpha_{B}$. As resulting to increase of coefficient with ferrite percentage increase at first, then coefficient amounting to maximum, and then it has decreased.

The inverse ME conversion factor $\alpha_{B}$ is proportional to product of piezoelectric and piezomagnetic effective modules and inversely proportional to composite compliance module. With increase of the ferrite percentage, on the one hand, there is increase of the piezoelectric module's effective value and decrease of the compliance module, that leading to increase of value $\alpha_{B}$, on the other hand, with increase of the ferrite percentage, there is decrease of the piezomodule, that leading to decrease of value $\alpha_{B}$. As a 
consequence of that, the value of coefficient is increase at first with increase of the ferrite percentage, is at the high and then is decrease. Similarly leading oneself the ME voltage coefficient. Its value also is proportionally to product of piezoelectric and piezomagnetic effective modules. However it is inversely not only to composite compliance module, but is inversely to product of compliance module and composite permittivity coefficient, that value also decrease with increase of ferrite concentration. This is leading to that the ME voltage coefficient maximum account to the most content of ferrite, than the inverse ME conversion factor maximum.

\section{References}

[1] I. B. Dzyaloshinskii, Zh. Eksp. Teor. Fiz. 37, 882 (1959).

[2] D. N. Astrov, Zh. Eksp. Teor. Fiz. 40, 1035 (1961).

[3] V.J. Folen, G.T. Rado, E.W. Stalder. Phys. Rev. Lett. 6, 607 (1961).

[4] E.A.Turov. Physics-Uspekhi 164, 325 (1994).

[5] Antonenkov O.V., Nikiforov I.S., Filippov D.A. Ferroelectrics. 279, 57 (2002).

[6] M. Fiebig. J. Phys. D: Appl. Phys. 38, 123 (2005).
[7] S. Dong, J.F. Li, D.Viehland, J. Cheng, L.E. Cross. Appl. Phys. Lett. 85, 3534 (2004).

[8] Y.K. Fetisov, V.M. Petrov, G. Srinivasan. J. Mater. Res. 22, 2074 (2007).

[9] A. A. Bush, K. E. Kamentsev, V. F. Meshcheryakov, Y. K. Fetisov, D. V. Chashin and L. Y. Fetisov, Zh. Tekh. Fiz. 79 (9), 71 (2009).

[10] Y.K. Fetisov, K.E. Kamentsev, D.V. Chashin, L.Y. Fetisov, G. Srinivasan. J. Appl. Phys. 105, 123918 (2009).

[11] D.A. Filippov, T.A. Galkina, G. Srinivasan. Pis'ma Zh. Tekh. 36, 21, 23 (2010).

[12] G. Harshe, J.O. Dougherty, R. E. Newnham. Int. J. Appl. Electromagn. Mater. 4. 145 (1993).

[13] G. Harshe, J.P. Dougherty, R.E. Newnham. Int. J. Appl. Electromagn. Mater. 4. 161 (1993).

[14] M.I. Bichurin, V.M. Petrov, G. Srinivasan. Phys. Rev. B. 68. 054402 (2003).

[15] M.I. Bichurin, V.M. Petrov, D.A. Filippov, V.M. Laletin, G. Srinivasan. Perspectivnye materialy №6, 5 (2004).

[16] W.P. Mazon. Phys. Rev. 74, 1134 (1948).

[17] D.A. Filippov, M.I. Bichurin, V.M. Petrov, V.M. Laletin, G. Srinivasan. FTT, 46, 9, 1621 (2004). 